\title{
Metallothionein 2 regulates endothelial cell migration through transcriptional regulation of vegfc expression
}

\author{
Annika Schuermann ${ }^{1,2}$ - Christian S. M. Helker ${ }^{1,3} \cdot$ Wiebke Herzog ${ }^{1,2,4}$
}

Received: 6 March 2015 / Accepted: 21 May 2015/Published online: 22 July 2015

(c) The Author(s) 2015. This article is published with open access at Springerlink.com

\begin{abstract}
Analysis of developmental angiogenesis can help to identify regulatory networks, which also contribute to disease-related vascular growth. Vascular endothelial growth factors (Vegf) drive angiogenic processes such as sprouting, endothelial cell (EC) migration and proliferation. However, how Vegf expression is regulated during development is not well understood. By analyzing developmental zebrafish angiogenesis, we have identified Metallothionein $2(\mathrm{Mt} 2)$ as a novel regulator of vegfc expression. While Metallothioneins (Mts) have been extensively analyzed for their capability of regulating homeostasis and metal detoxification, we demonstrate that $\mathrm{Mt} 2$ is required for EC migration, proliferation and angiogenic sprouting upstream of vegfc expression. We further demonstrate that another Mt family member cannot compensate Mt2 deficiency and therefore postulate that $\mathrm{Mt} 2$ regulates angiogenesis independent of its canonical Mt function. Our data not only reveal a non-canonical function of Mt2 in angiogenesis, but also propose Mt2 as a novel regulator of vegfc expression.
\end{abstract}

Electronic supplementary material The online version of this article (doi:10.1007/s10456-015-9473-6) contains supplementary material, which is available to authorized users.

\footnotetext{
Wiebke Herzog

wiebke.herzog@mpi-muenster.mpg.de

University of Muenster, Muenster, Germany

2 Cells-in-Motion Cluster of Excellence (EXC 1003 - CiM), University of Muenster, Muenster, Germany

3 Present Address: Max-Planck-Institute for Heart and Lung Research, Bad Nauheim, Germany

4 Max-Planck-Institute for Molecular Biomedicine, Muenster, Germany
}

Keywords Vegfc $\cdot$ Angiogenesis $\cdot$ Endothelial cell migration - TALEN · Nonsense-mediated decay . Phenotype variability $\cdot$ Zebrafish

\section{Introduction}

Growth of blood vessels during development as well as in the adult organism is a tightly regulated process, which is controlled by endothelial cell (EC) behaviors such as cell migration, proliferation and differentiation. Misregulation of vascular growth not only contributes to ischemic conditions, but overgrowth also directly aggravates diseases such as growth and metastasis of cancers or age-related macular degeneration.

Vascular endothelial growth factors (Vegfs) and their Vegf receptors (VEGFR-1/Flt1, VEGFR-2/Kdrl-Kdr and VEGFR-3/Flt4) are the major regulators of vascular growth processes [1-3].

While VEGFA and VEGFR-2 (Kdrl in zebrafish) mainly regulate angiogenic processes such as sprouting and remodeling of vessels [4, 5], VEGFC and VEGFR-3 have mainly been recognized for their role in regulating development of the lymphatic endothelial system [6-8]. Vegfr3/ ftt-deficient zebrafish completely lack lymphatic vessels but show no major defects in blood vessel growth [9]. VEGFR3-deficient mice die of defective vascular development before the lymphatic system becomes established [10]. Vegfc mutant mice as well as zebrafish lack a lymphatic system [11, 12]. Angiogenesis defects observed in vegfc zebrafish mutants include failure in EC migration [during formation of the primordial hindbrain channels (PHBCs)] [11] as well as reduced EC proliferation [in the common cardinal veins (CCVs)] [13]. 
However, VEGFA as well as VEGFC expression are both upregulated in various tumors and their misregulation is involved in other diseases; therefore, understanding the mechanisms regulating their expression are of clinical relevance [14, 15].

Within cultured fibroblast or cancer cells, VEGFC mRNA expression was shown to be upregulated by cytokines (Interleukin- $1 \alpha$ or interleukin- $1 \beta$, or Tumor necrosis factor- $\alpha$ ) [16] and growth factors (Platelet derived growth factor, Epidermal growth factor and Transforming growth factor- $\beta$ ) [17], but not by Hypoxia-inducible factor-1 $\alpha$ (HIF1 $\alpha)$ [18].

The optical clarity of the externally developing zebrafish embryos is one of the many advantages for using this model for the analysis of vascular development. The growing vasculature can easily be visualized in vivo by endothelial-specific transgenic fluorophore expression [19].

The vascular anatomy of zebrafish embryos has a high structural homology to other vertebrates [20, 21]. Similarly, most signaling pathways regulating vascular development are conserved between zebrafish and mammals [22, 23]. A functional circulatory system including a primitive heart is already established in the zebrafish embryo by $24 \mathrm{~h}$ postfertilization (hpf).

Additionally, recent advances in genome editing using Transcription activator-like effector nucleases (TALENs) or Cas9 nucleases [24, 25] enabled gene-specific targeting in zebrafish.

We performed gene expression analyses to identify novel regulators of angiogenesis in zebrafish embryos and thereby identified metallothionein $2(m+2)$ as a candidate.

MTs are low-molecular-weight and cysteine-rich proteins, which are conserved throughout the animal kingdom. There are four classes of mammalian $M t$ genes, Mt1-4 [26, 27], and two $m t$ genes in zebrafish, $m t 2$ and metallothionein-B-like ( $m t b l)$ [28, 29].

The main function of MTs is the regulation of homeostasis, such as the protection against oxidative stress or metals. Both heavy and trace metals such as zinc, copper or iron can be chelated via sulfur-based clusters [30, 31].

However, MTs also display non-canonical functions in angiogenesis and pathological conditions. $M t 1$ and $M t 2$ are very similar and the best characterized genes of the MT family, which can act as tumor suppressors [32] and have cardio- and neuroprotective functions [33-35]. Mice deficient for both $M t 1$ and $M t 2$ are viable and show beside their greater sensitivity to metals no major developmental defects [36, 37]. When challenged by femoral artery ligation or cortical freeze injury, these $M t 1 / 2$ knockout mice show impaired angiogenesis and wound healing [38-40]. MT3 is important for cell growth [41], and its expression is downregulated in a carcinoma cell line [42]. MT3 also has a critical role in the recovery of the brain, since Mt3-deficient mice show increased oxidative stress and apoptosis upon cortical freeze lesion [43]. The non-inducible $M t 4$ is expressed in epithelial tissues and has only been shown to detoxify of metals [30, 44].

However, how MTs exert their non-canonical functions, such as the regulation of angiogenic processes, is not understood.

Here, we used zebrafish as a model to analyze the role of Mt in angiogenesis. We generated Mt2-deficient zebrafish embryos by performing antisense morpholino-oligonucleotide (MO)-mediated gene knockdown as well as by using TALEN to generate zebrafish $m t 2$ mutants. Using in vivo time-lapse analysis, we show that $m t 2$ deficiency leads to striking angiogenesis defects, especially to defective formation of the PHBCs. Furthermore, we demonstrate that $\mathrm{Mt} 2$ acts upstream of vegfc expression in regulating EC migration and proliferation. This regulation of angiogenesis represents a non-canonical function of $\mathrm{Mt} 2$, since another Metallothionein family member (Mtbl) cannot regulate vegfc expression.

\section{Materials and methods}

\section{Zebrafish maintenance and strains}

Zebrafish embryos were maintained at $28.5^{\circ} \mathrm{C}$ under standard husbandry conditions [45]. Zebrafish lines used were $\operatorname{Tg}(k d r l: E G F P)^{s 843}$ [46], $T g(f l i l a: E G F P)^{y 1}$ [47] and Tg(flila:nEGFP $)^{y 7}$ [48]. The vegfc ${ }^{\text {hu6410 }}$ allele encodes a stop codon at amino acid position 107 (L107X) [49].

\section{Generation of the $m+2$ mutant transgenic zebrafish line using transcription activator-like effector nucleases (TALENs)}

TALENs were assembled using the Golden Gate method [50]. For targeting the $m t 2$ locus, a $5^{\prime}$ RVD (NH-NG-NHNH-NI-NG-NI-HD-NG-HD-NG-HD-NG-NH (DNA sequence GTGGATACTCTCTGG)) and a $3^{\prime}$ RVD (NIHD-NG-HD-NG-NG-NH-NH-HD-NI-HD-NI-NG-NG (DNA sequence ACTCTTGGCACATTC)) were generated with a spacer of $16 \mathrm{bp}$ (AAAAATGGACCCCTGC) to target exon 1. An AvalI (New England BioLabs) restriction site within the spacer region was used for genotyping of putative founders. mRNA was generated using the T3 mMessage mMachine Kit (Ambion) and injected using $100 \mathrm{pg}$ of the TALEN mix.

\section{mRNA and morpholino (MO) injections}

MOs blocking either translation (MO) or RNA splicing (spbMO) were obtained from Gene Tools and are as follows: 
$m t 2$ MO: 5'-GGTCCATTTTTCCAGAGAGTATCCT (5 ng) and $m t 2$ spbMO: 5'-AGCTGAAACACTTACTCTT GGCACA (7-10 ng), targeting $m t 2$ (BC152694.1); $m t b l$ MO: 5'-CTGGTCCATCTTTACACCGTAGGTC and $m t b l$ spbMO: 5'-AGTTAATCGGCTCACTTTTCTTGTC (both 13 ng) targeting mtbl (NM_001201469.1), upfl spbMO: 5' TTTTGGGAGTTTATACCTGGTTGTC (0.1 ng) [51] and smgl spbMO: 5'-AACCATTGGTTTGTTACCTGGTGCA (12.5 ng) [51] and standard control MO: 5'-CCTCTTACC TCAGTTACAATTTATA.

For overexpression experiments, the $m t 2$ sequence was amplified from $24 \mathrm{hpf}$ cDNA and cloned into the pCS2+ vector for in vitro RNA synthesis using the following primers: $m t 2 f w d$ 5'-AGACGAATTCGCTCCACCATG GACCCCTGCGAATGTGC and $m t 2 r e v 5^{\prime}$-AGACCTCGA GTCATTGACAGCAGCTGGAGC.

Similarly, $m t b l$ was cloned into the pCS2+ vector using mtblfwd 5'-AGACGAATTCGCTCCACCATGGACCAGT GTAACTGCTC and mtblrev 5' - AGACCTCGAGTCATT TGCAGCAGTGTGTGG.

The mRNA was synthesized using SP6 mMessage mMachine Kit (Ambion). For all experiments, the injection was done into the yolk of 1-cell-stage zebrafish embryos, and $0.05 \%$ phenol red (Sigma) was added to the injection solution.

Injection amounts per embryo were as follows: $500 \mathrm{pg}$ $m t 2$ mRNA, $100 \mathrm{pg} m t b l$ mRNA, $100 \mathrm{pg}$ to $500 \mathrm{pg} \mathrm{H} 2 \mathrm{~B}$ cherry mRNA [52], 200 pg vegfc mRNA [53] and 200 pg sFLT4 mRNA [54].

\section{RNA and DNA isolation, qPCR analysis and genotyping}

RNA from WT, mutants and MO-injected embryos was isolated with Trizol reagent, and cDNA was generated by SuperScript II reverse transcriptase (Invitrogen).

The cDNA was analyzed with real-time quantitative PCR (qPCR) using Power SYBR Green (Applied Biosystems) and the following primers: b-actinfiwd $5^{\prime}$-CTGGAC TTCGAGCAGGAGAT and b-actinrev 5'-GCAAGATTC CATACCCAGGA (156 bp amplicon); vegfcfwd 5'-GCAG GAACATCAGCACTTCA and vegfcrev 5'-GTGTGGTTG GCGAAGCTTAT (103 bp amplicon); flilafwd 5'-CTCAG GGAAAGTAGCTCATCG and filarev 5'-CTTTTCCGC TGTGCATGTT (139 bp amplicon); myodlfwd 5'-TCTGA TGGCATGATGGATTT and myodlrev 5'-TTATTA TTCCGTGCGTCAGC (110 bp amplicon). For qPCR at least two different cDNA samples were generated and analyzed. Experiments were performed at least three times.

The knockdown efficiency of the $m t 2$ splice MO was validated with reverse transcription PCR (RT-PCR) and primers $m t 2 f w d 5^{\prime}$-ATGGACCCCTGCGAATGTGC and $m t 2 r e v \quad 5$-TCTTCTTGCAGGTAGTACACTG (spliced amplicon $91 \mathrm{bp}$, non-spliced amplicon $185 \mathrm{bp}$ ). The functionality of the $m t b l$ splice MO was analyzed with mtblfwd 5'-GACCAGTGTGACTGCTCCAA and mtblrev 5'-TGCA GGATTTCTCCTTGTCC (spliced amplicon 169 bp, nonspliced amplicon $327 \mathrm{bp}$ ).

DNA was extracted using lysis buffer (10 mM Tris$\mathrm{HCl}, 50 \mathrm{mM} \mathrm{KCl}, 0.3 \%$ Tween 20, $0.3 \%$ Nonidet P-40, $\mathrm{pH} 8.3$ ) with $0.5 \mu \mathrm{g} / \mu \mathrm{l}$ proteinase $\mathrm{K}$ (Roche) overnight at $55^{\circ} \mathrm{C}$, followed by $10 \mathrm{~min}$ denaturation at $95{ }^{\circ} \mathrm{C}$.

The genotype of the $m t 2^{m u 290}$, the $m t 2^{m u 292}$ and the $m t 2^{m u 289}$ mutants was analyzed with primers $m t 2 f w d 5^{\prime}-\mathrm{T}$ CTTCTTGCAGGTAGTACACTG and $m t 2 r e v$ 5'-TAAAA GCAGAGCACAAACACG and the restriction enzyme AvalI.

The genotype of the vegfc ${ }^{h u 6410}$ zebrafish mutants was analyzed in a multiplex PCR with WT and mutant zebrafish-specific inner and outer primers. As inner primers vegfffwd 5'-CTTTCATCAATCTTGAACTTTT (WT specific) and vegfcrev 5'-TAAATTAATAGTCAC TCACTTTACT (mutant specific with one mismatch) were used and as outer primers vegfcfwd $5^{\prime}$-GATGAACTCATG AGGATAGTTT and vegfcrev 5'-AAACTCTTTCCCCAC ATCTA.

\section{Whole-mount in situ hybridization}

Whole-mount in situ hybridization was performed as described [55]. The $m t 2$ probe was amplified from $24 \mathrm{hpf}$ zebrafish embryo cDNA with the $\mathrm{T} 7$ promoter site and the following primers: $m t 2 f w d 5^{\prime}$-GGAACTTTCAAGCTCTT TGTGG and $m t 2 r e v 5^{\prime}$-gTAATACgACTCACTATAggGA CAAAGGACATGGCAGAAAA. The vegfc probe is already described [53].

\section{Confocal microscopy and in vivo time-lapse analysis}

Zebrafish embryos were analyzed with confocal microscopy as previously described, using $1 \%$ agarose embryo moulds [56]. The fluorescent images were acquired using the Sp5 DM 6000 upright confocal microscope (Leica) or the inverse LSM 780 confocal microscope (Zeiss).

\section{BrdU incorporation and immunohistochemistry}

Proliferation analysis was performed as described [57] with following modifications: Embryos were grown to $24 \mathrm{hpf}$ and then incubated in $10 \mathrm{mM}$ 5-bromo- 2 '-deoxyuridine (BrdU) for $30 \mathrm{~min}$ on ice. After $8 \mathrm{~h}$ of further incubation and BrdU incorporation, embryos were fixed in $4 \%$ paraformaldehyde (PFA) at $32 \mathrm{hpf}$. After incubation in $2 \mathrm{M} \mathrm{HCl}$ for $1 \mathrm{~h}$, permeabilization (phosphate-buffered saline (PBS) with $0.3 \%$ Triton $\mathrm{X}-100$ (Sigma) and $0.1 \%$ Tween 20 (Sigma)) and blocking (PBS with $0.3 \%$ Triton 
$\mathrm{X}-100$ and $4 \%$ BSA (Roth)), the following antibodies were used for immunostaining: mouse anti-BrdU (1:100, Roche), Alexa 546 anti-mouse (1:500, Invitrogen) and Alexa 488 anti-GFP (1:500, Invitrogen, for ECs of $\left.T g(k d r l: E G F P)^{s 843}\right)$. After each antibody incubation, extensive washing was performed (PBS with $0.3 \%$ Triton $\mathrm{X}-100)$.

\section{Phenotypic analysis, quantifications, statistics and softwares}

For evaluation of the rescue experiments, different clutches of at least three different experiments were scored for the existence of the PHBCs. If the PHBCs were not present at all or developed to $<50 \%$, they were considered as missing; if the PHBCs were developed to more than $50 \%$ or fully connected, they were considered as existent. For rescue experiments of vegfc ${ }^{h u 6410}$ zebrafish mutants, only embryos with a strong PHBC phenotype or with fully developed PHBCs were taken for analysis for both Ctr and $m t 2$ mRNA-injected zebrafish, and each embryo was subjected to subsequent genotyping. Cell numbers of fixed $T g(k d r l: E G F P)^{s 843}$ or $T g(\text { flila: } n E G F P)^{y 7} \quad$ zebrafish embryos were evaluated with help of the Spots function of Imaris. Cells in the PHBC, the anterior cluster and the posterior clusters were counted at $32 \mathrm{hpf}$ in confocal stacks. Similarly, ECs of the ACV, PCV and CCV were counted at $32 \mathrm{hpf}$, while ECs of the Ses were counted at 48 hpf.

For analysis of the amount of proliferating cells in the $\mathrm{CCVs}$, BrdU-positive cells were calculated relative to the total number of ECs in the CCVs. For quantifying the Ses, the region between somites 9 and 14 has been analyzed. The $P$ values for the experiments were calculated with a two-tailed Student's $t$-test. The rescue experiments for the PHBC phenotypes were evaluated for significance with the Chi-Square test using Microsoft excel. SDS 2.3 and RQ Manager (Applied Biosystems) were taken for analysis of the real-time data. Primers were designed using Primer3 (http://bioinfo.ut.ee/primer3-0.4.0/primer3/input.htm).

Where possible, the analysis was performed blind to experimental conditions.

\section{Results}

\section{Mt2 regulates EC behavior during angiogenesis}

To identify regulators of EC migration, we screened for functional involvement of candidate genes using morpholino antisense oligonucelotides (MO) to knockdown protein expression in zebrafish embryos and analyzed their vascular development using endothelial-specific GFP expression $\left(T g(k d r l: G F P)^{s 843}\right)$.

We identified Mt2 as a potential regulator of EC migration. For a detailed analysis, we injected MOs either inhibiting mRNA translation (using MO covering the ATG) or blocking mRNA splicing (spbMO). Embryos injected with $m t 2 \mathrm{MO}$ or $m t 2 \mathrm{spbMO}$, showed brain necrosis but no other major morphological defects (Fig. 1). Of the affected vessels, the PHBCs are the first to develop. They grow by angiogenic sprouting out of an anterior cluster and a posterior cluster of ECs, which start at $18 \mathrm{hpf}$ to migrate toward each other and connect around 22-23 hpf to form a functional vessel (Fig. 1d, e; movie 1). At 24-25 hpf, circulation starts and blood flow can be observed going through the PHBCs. However, we observed not only defective growth of the PHBCs, but also of the CCVs and the Ses at different time points of development (Fig. 1, Fig. S1).

We used in vivo time-lapse imaging to further analyze PHBC formation in control MO (Ctr)- or $m t 2 \mathrm{MO}$-injected $T g(k d r l: E G F P)^{s 843}$ embryos. The Ctr and $m t 2$ MO-injected (morphant) embryos were indistinguishable from each other until $18 \mathrm{hpf}$ (Fig. 1e, f), with both displaying normal development of the lateral dorsal aorta (LDA). In Ctr morphants, the ECs migrated, connected and thereby formed the PHBCs (Fig. 1e, movie 1), whereas $m t 2$ morphant ECs failed to migrate out of the clusters and did not connect to form the PHBC (Fig. 1f, movie 2). However, the ECs were motile and formed filopodia, but the directed migration required for the connection of the PHBCs was perturbed (movie 2). Some $m t 2$-deficient embryos extended sprouts from the anterior and posterior cluster to develop the PHBCs, but no proper connection was established. To determine, whether this defect is caused by defective migration or reduced EC numbers, we counted the number of ECs in the PHBCs as well as in the anterior and posterior cluster at $32 \mathrm{hpf}$, long after PHBC formation should have been completed. While the total EC number in PHBCs and clusters was similar, $\mathrm{Ctr}$ morphants had an average of 21 cells in the PHBC and 12 cells in the clusters, whereas $m t 2$ morphants had an average of 13 cells in the PHBC and 22 cells in the cluster (Fig. 1g). Therefore, our results indicate that $\mathrm{Mt} 2$ regulates $\mathrm{EC}$ migration during $\mathrm{PHBC}$ angiogenesis.

Additionally, we analyzed CCV formation in Ctr and $m t 2$ morphants in more detail (Fig. S1). The CCVs grow at a $90{ }^{\circ} \mathrm{C}$ angle out of the trunk ACV and posterior PCV, by a combination of collective EC migration and proliferation [13]. At 32 hpf the total cell number in ACV, PCV and CCV was reduced in $m t 2$ morphants compared with Ctr morphants (Fig. S1j). However, the percentage of cells in the $\mathrm{CCV}$ was significantly reduced from $35 \% \mathrm{CCV}$ cells in $\mathrm{Ctr}$ embryos to $26 \%$ in mt2-deficient embryos (Fig. S1i). To test whether Mt2 regulates EC migration or proliferation in 


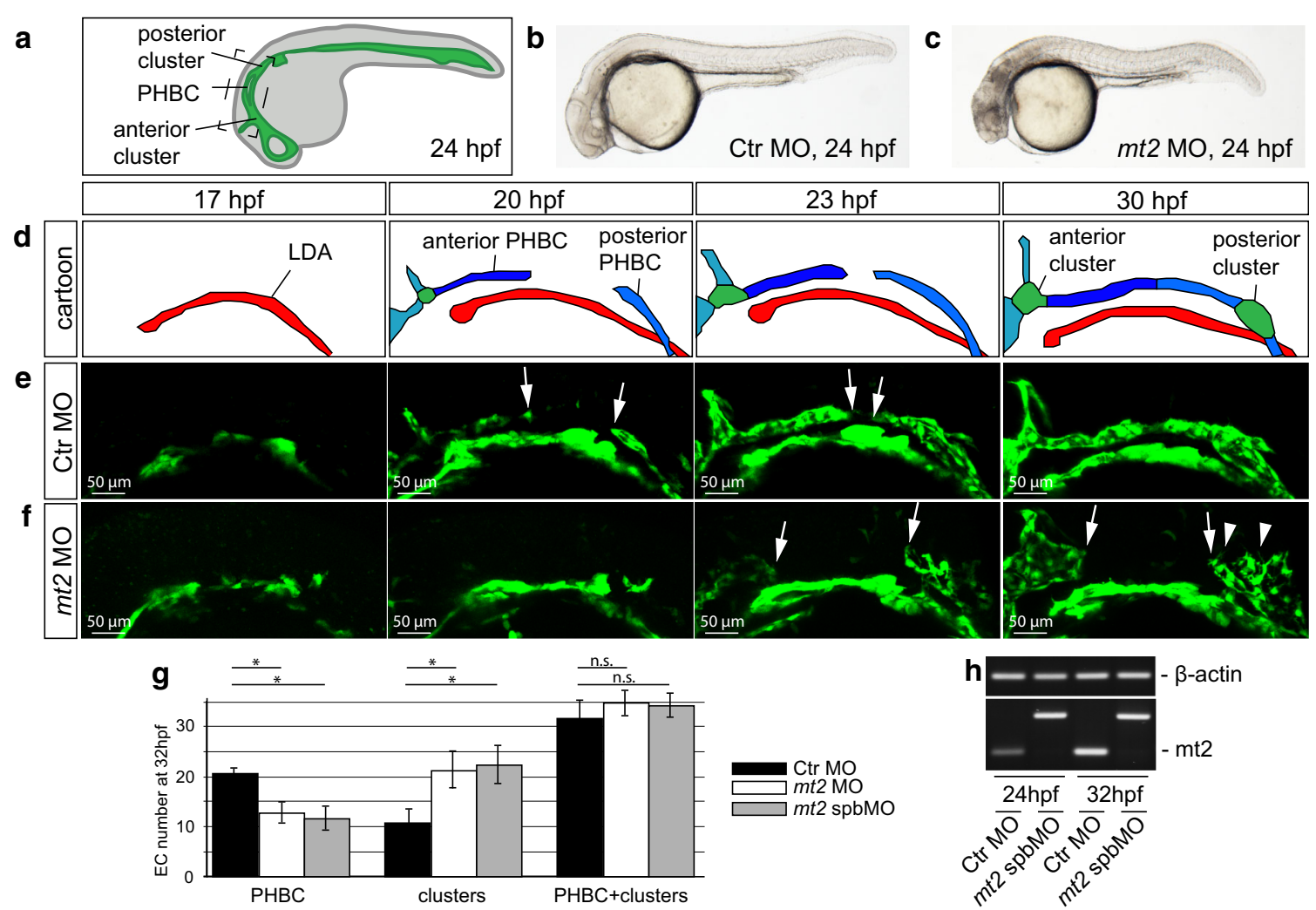

Fig. $1 m t 2$ morphants fail to form the PHBCs. a Schematic illustration of the vasculature of a $24 \mathrm{hpf}$ old zebrafish embryo with the location of the PHBC and the adjacent PHBC forming clusters. $\mathbf{b}, \mathbf{c}$ Brightfield images of $\mathrm{Ctr}$ (b) and $m t 2$ (c) MO-injected zebrafish embryos at $24 \mathrm{hpf}$ showed no morphological defects, apart from a mild necrosis in the head. $\mathbf{d}$ Schematic illustration of the development of the PHBC (dark blue) between 17 and $30 \mathrm{hpf}$. e, f Confocal micrographs from time-lapse movies showing the development of the PHBC in zebrafish embryos between 18 and $30 \mathrm{hpf}$. The vasculature was visualized by transgenic GFP expression using $T g(k d r l: E G F P)^{s 843}$ embryos. In embryos injected with Ctr MO, ECs migrate from the anterior and the posterior cluster and connect to form the PHBC before $24 \mathrm{hpf}$ (at around $23 \mathrm{hpf}$; e). In embryos injected with $m t 2 \mathrm{MO}$ ECs fail to migrate and therefore do not form

the $\mathrm{CCV}$, we performed proliferation analysis by $\mathrm{BrdU}$ incorporation in $m t 2$ morphants. Proliferation was strongly decreased in $m t 2$ morphants (Fig. S2).

In sum, our results indicate that $\mathrm{Mt} 2$ regulates angiogenesis, by regulating EC migration in the PHBCs and EC proliferation during $\mathrm{CCV}$ formation.

\section{$m t 2$ zebrafish mutants phenocopy the $m t 2$ morphants}

Despite performing extensive control experiments, MOs have been shown to exhibit off-target effects [58-60]. To verify the phenotype of the $m t 2$ morphants, we used TALENs [50] to induce double-strand breaks in the $m t 2$ gene. As expected, errors made by the repair machinery of the cell then led to mutations in the double-strand the PHBCs (f). White arrows indicate the anterior and posterior migration front of the PHBC. White arrowheads indicate filopodia in $m t 2$ morphants. g Quantification of EC numbers in Ctr MO-injected (black bars) or $m t 2 \mathrm{MO}$-injected (white bars) and $m t 2$ spbMOinjected (gray bars) embryos counted from vascular-specific nuclear GFP expression $\left(\operatorname{Tg}(f i 1 a: n E G F P)^{y 7}\right)$. While the total EC numbers were not affected, $m t 2$ MO-injected embryos showed fewer ECs in the PHBC and more ECs in the clusters. $n=20, * P<0.05$; n.s., not significant; error bars indicate standard error of the mean (SEM). h Analysis of $m t 2$ splicing efficiency in embryos injected with $\mathrm{Ctr}$ MO or $m t 2$ spbMO. RT-PCR analysis showed a $185 \mathrm{bp}$ amplicon in embryos injected with $m t 2$ spbMO, while functional splicing led to a 91 bp amplicon in Ctr MO-injected embryos. (Color figure online)

break area [50]. Since the $m t 2$ sequence is very short, we targeted exon 1, which consists of 25 base pairs (bp) only (Fig. 2a). We identified several different alleles of $m t 2$ mutations and further analyzed three of them.

In the $m t 2^{m u 289}$ mutant allele only 6 bp were deleted, which resulted in deletion of the second and third amino acid of the Mt2 protein (Fig. 2b, S3). The $m t 2^{m u 290}$ sequence has two-point mutations and an insertion of $8 \mathrm{bp}$, which led to a frame shift and an early stop codon. The $m t 2^{m u 292}$ sequence has a deletion of $15 \mathrm{bp}$, which lead to a frame shift and an early stop codon (Fig. 2b, S3). Since the mutations of $m t 2^{m u 290}$ and $m t 2^{m u 292}$ are located next to the start codon and there is no downstream start codon in frame, the original Mt2 protein sequence should be completely lost, supposedly resulting in null mutants. 


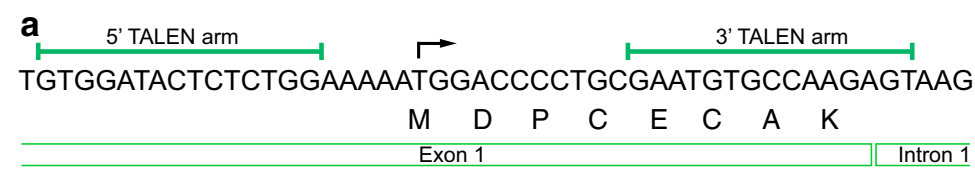

b

protein sequence WT Mt2: mdpcecaktg tcncgatckc tncqcttckk sccsccpsgc skcasgcvck gnscgssccq protein sequence Mt2mu289: m--cecaktg tcncgatckc tncqcttckk sccsccpsgc skcasgcvck gnscgssccq protein sequence Mt2mu290: mcqvislrmc qdwnlqlwcy lqvh protein sequence Mt2mu292: mcqdwnlqlw cylqvh
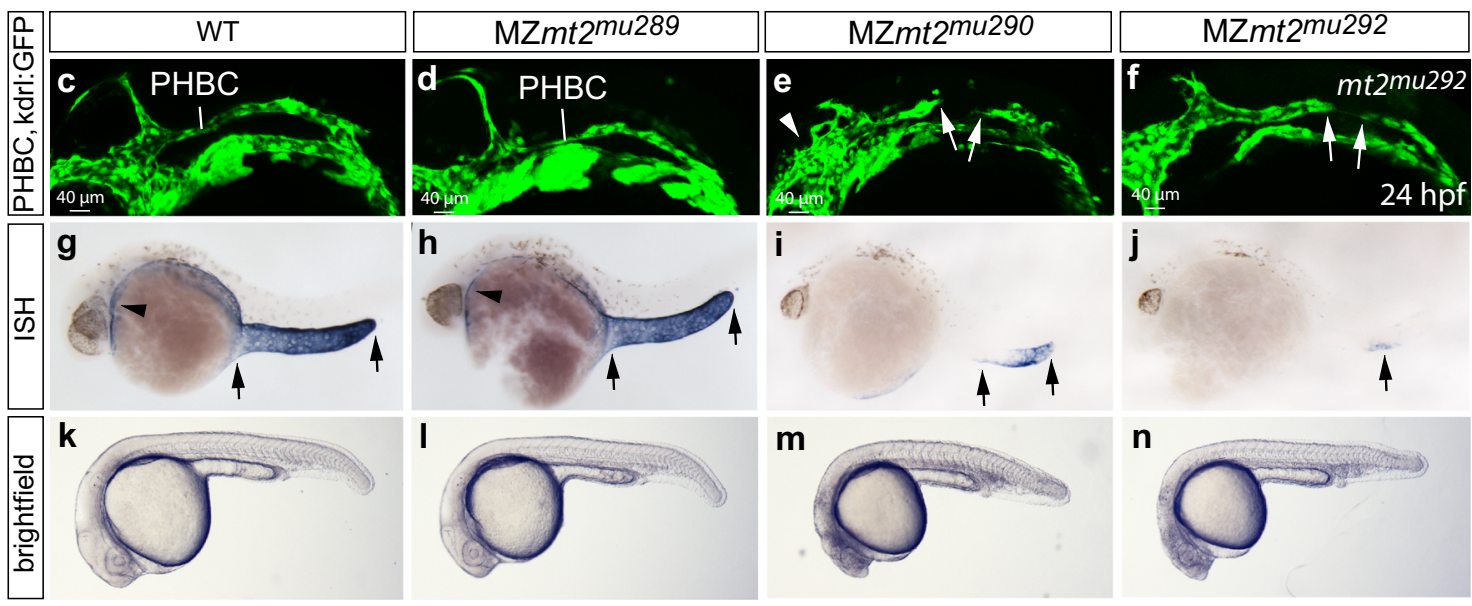

Fig. 2 TALEN-generated $m t 2$ zebrafish mutants fail to form the PHBC and have different levels of NMD of $m t 2$ transcripts. a $5^{\prime}$ and $3^{\prime}$ TALEN arms were designed to target exon 1 of the $m t 2$ gene to induce mutations in the genome. b TALEN injection resulted in various genomic mutations. Illustrated is the comparison of the amino acid sequence in WT and different Mt2 mutant alleles, red color indicates mutated amino acids. In $m t 2^{m u 289}$ mutants a 6 bp deletion resulted in deletion of two amino acids, while in $m t 2^{m u 290}$ and $m t 2^{m u 292}$ frameshift mutations resulted in complete changes of the amino acid sequence. c-f Confocal images of the PHBCs at $24 \mathrm{hpf}$. WT (c) and MZmt $2^{m u 289}$ mutant zebrafish embryos (d) form a PHBC, while MZmt $2^{m u 290}$ and $M Z m t 2^{m u 292}$ mutant embryos fail to connect the PHBCs. The vasculature was visualized by transgenic GFP expression from $\operatorname{Tg}(\text { flila:EGFP })^{y l}$ for $\mathrm{MZ} m t 2^{m u 289}$ and $\mathrm{MZmt2} 2^{m u 290}$

By mating F1 heterozygous carriers of each $m t 2$ allele, we obtained homozygous F2 embryos. To our surprise, we only detected very weak phenotypes (data not shown). Since $m t 2$ is maternally provided [61], we hypothesized that the maternal mRNA is sufficient to rescue $m t 2$ deficiency during the early developmental stages analyzed. Therefore, we raised homozygous F2 embryos to adulthood. When mating homozygous $m t 2$ mutant fish to obtain maternal and zygotic mutant (MZ) F3 offspring, we observed strong morphological and angiogenesis phenotypes (Fig. 2), phenocopying the $m t 2$ morphants. Both

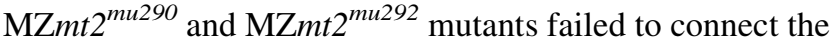
PHBCs, had reduced cell numbers in the CCVs and defective Ses formation. (Fig. 2e, f; Fig. S4). Additionally, in a subset of $m t 2$ mutant embryos the morphology of the PHBC clusters was affected, with the ECs forming ectopic sprouts (arrowhead, Fig. 2e). The MZmt2 ${ }^{m u 289}$ zebrafish mutant embryos and from $T g(k d r l: E G F P)^{s 843}$ for MZmt $2^{m u 292}$ mutant embryos. White arrows indicate the anterior and posterior migration front of the PHBC. $m t 2$ expression was analyzed by in situ hybridization in 24 hpf-old embryos. While WT siblings (g) and MZmt $2^{m u 289}$ mutants (h) had similar $m t 2$ expression levels, nonsensemediated decay led to degradation of $m t 2$ mRNA transcript in MZmt $2^{m u 290}$ (i) and MZmt $2^{m u 292}$ (j) mutant embryos. Black arrows indicate $m t 2$ expression in cells of the yolk extension, black arrowheads label the region of the PHBCs. $\mathbf{k}-\mathbf{n}$ Brightfield images of WT, MZmt2 $2^{m u 289}$, MZmt2 $2^{m u 290}$ and MZmt $2^{m u 292}$ mutant embryos at $24 \mathrm{hpf}$. WT siblings and MZmt $2^{m u 289}$ displayed no morphological defects. MZmt $2^{m u 290}$ and MZmt $2^{m u 292}$ mutant embryos are smaller in size and display necrosis in the head

mutants, which lack only two amino acids, displayed only a very weak phenotype. The PHBCs (Fig. 2d) and the Ses (Fig. S4g) developed normally in those mutants, while a mild phenotype could be observed in the CCVs (Fig. S4b).

The penetrance and severity of the phenotype for both null mutants were variable within the clutch and between clutches. MZmt $2^{m u 290}$ zebrafish mutants showed severe phenotypes at higher rates than MZmt $2^{m u 292}$ zebrafish mutants (compare Table 1), although both should not retain any amino acid sequence of $\mathrm{Mt} 2$. In order to investigate whether the mutations were causing strong alleles, we examined the level of gene transcription. One mechanism potentially interfering with mRNA transcript stability in mutants is nonsense-mediated decay (NMD), whereas MO-mediated blocking of translation would rather stabilize the transcript.

We therefore subjected 24 hpf-old MZmt2 mutant embryos to in situ hybridization to analyze the presence of 
Table $1 m t 2$ zebrafish morphants, MZmt2 zebrafish mutants and vegfc ${ }^{\text {hu6410 }}$ zebrafish mutants display many common phenotypes

\begin{tabular}{lccrr}
\hline & PHBCs & Clusters & \multicolumn{1}{c}{ Ses } & CCVs \\
\hline WT & 0 & 2.26 & 2.53 & 4.75 \\
$m t 2$ MO & 79.07 & 92.78 & 94.29 & 84.76 \\
$m t$ spbMO & 75.00 & 85.71 & 90.24 & 77.78 \\
MZmt $2^{\text {mu289 }}$ & 0 & 3.64 & 6.50 & 18.20 \\
MZmt2 $^{\text {mu290 }}$ & 34.22 & 84.42 & 90.37 & 41.63 \\
MZmt2 $^{\text {mu292 }}$ & 8.02 & 35.73 & 68.75 & 26.78 \\
vegfc $^{\text {hu6410 }}$ & 59.52 & 7.50 & 6.34 & 65.00 \\
\hline
\end{tabular}

Overview of the frequencies of the different phenotypes observed upon $m t 2$ deficiency compared to $v e g f c$ deficiency and WT zebrafish embryos. The following classification of phenotypes was scored as affected: PHBCs: The PHBCs were developed to less than $50 \%$ in length; cluster: severely thickened anterior cluster or additional ectopic sprouts or holes; CCVs: reduction by more than $15 \%$ of EC numbers; Ses (scored between somites 9 and 14): Se sprouts were either significantly shortened by more than $15 \%$ or Se numbers were reduced to less than $85 \%$. The PHBC, cluster and Se phenotypes were analyzed at $24 \mathrm{hpf}$; the CCVs were analyzed at $32 \mathrm{hpf}$ (WT $n=138$, vegfc $^{h u 6410} n=93, m t 2 \mathrm{MO} n=168, m t 2 \operatorname{spbMO} n=157$, $m t 2^{m u 292} n=256, m t 2^{m u 290} n=128, m t 2^{m u 289} n=123$ )

the $m t 2$ transcript. While WT and MZmt $2^{m u 289}$ mutant embryos expressed $m t 2$ as published [61], almost no expression could be observed in MZmt2 $2^{m u 290}$ and MZmt2 $2^{m u 292}$ mutants (Fig. 2g-j). Interestingly, the efficiency of NMD was not the same for both null mutants. While the great majority of MZmt $2^{m u 292}$ embryos completely lacked $m t 2$ expression (Fig. 2j), some MZmt $2^{m u 290}$ mutant embryos retained $m t 2$ message partially (Fig. 2i), which correlated with the different frequencies of angiogenesis defects (Table 1). We hypothesized that the more efficient the NMD was for the $m t 2$ zebrafish mutant, the more compensation mechanisms might take place to attenuate the phenotype. To analyze, whether mRNA stability could indeed influence the phenotypic severity, we partially ablated two subunits of the NMD mediating complex ( $\operatorname{smgl}$ and upfl) by injecting smg 1/upf1 MOs in WT and in MZmt $2^{m u 290}$ mutant embryos. We could indeed observe an increase in the number of affected embryos, when message degradation by NMD was reduced (Fig. S5). This indicates that indeed the correlation of the stronger phenotype with the reduced mRNA degradation is functionally relevant. The sum of this data implies that different levels of mRNA degradation can lead to differences in the phenotypes of generated zebrafish mutants and morphants, potentially by regulating unknown compensatory mechanisms.

\section{Mt2 acts upstream of Vegfc in regulating angiogenesis}

Mt2 deficiency resulted in angiogenic defects during PHBC and $\mathrm{CCV}$ formation. Both of these processes have been described to be regulated by Vegfc during zebrafish embryonic development. Vegfc mutants or morphants fail to connect the PHBCs and have reduced proliferation in their CCVs [11, 13].

We therefore carried out different rescue experiments to analyze whether there is an interaction of Mt2 and Vegfc signaling. We ubiquitously overexpressed Vegfc in WT or $m t 2$ morphant embryos by injection of vegfc mRNA into 1-cell-stage embryos. Overexpression of vegfc in WT embryos did not alter EC migration to form the PHBCs (Fig. 3a), but significantly reduced the number of embryos with PHBC connection defects from $44 \%$ affected $m t 2$ morphants to $25 \%$ affected vegfc-injected $m t 2$ morphants (Fig. 3c, j, n). Furthermore, we overexpressed the Vegfc ligand trap sft4 [54], which is a soluble form of the Vegfr3, that titrates away Vegfc and therefore results in the same phenotypes as the genetic vegfc mutation (Fig. 3e, k). By combining sflt 4 with high amounts of $m t 2$ mRNA injection, we could compensate the PHBC formation failure (Fig. 3f, $\mathrm{k}, \mathrm{o}$ ). Injection of vegfc mRNA rescued the sflt4 mRNA injection to a similar extent (data not shown), indicating that Mt2 overexpression could indeed compensate for Vegfc ligand depletion. Interestingly, when we repeated the same experiment of rescuing Vegfc deficiency by $m t 2$ overexpression in vegfc ${ }^{\text {hu6410 }}$ mutant embryos, Mt 2 failed to rescue (Fig. 3i, 1, p), suggesting that vegfc is the only relevant target of $m t 2$. We confirmed the upregulation of vegfc transcripts after $m t 2$ mRNA injection in vegfc ${ }^{h u 6410}$ mutant embryos with qPCR (Fig. S6). Taken together our data showed that Mt2 deficiency can be overcome by Vegfc addition and that Mt2 overexpression can outcompete Vegfc protein depletion, but not Vegfc mutation. These results are consistent with a mechanism, in which $\mathrm{Mt} 2$ regulates vegfc RNA expression (Fig. $3 \mathrm{~m}$ ).

\section{Mt2 regulates transcript levels of $v e g f c$}

Given the results above, we used qPCR to analyze vegfc transcript levels in $m t 2$ morphant, MZmt $2^{m u 290}$ mutant and $m t 2$-overexpressing embryos. qPCR analysis revealed a $20 \%$ decrease in vegfc RNA in $m t 2$ morphants and a $31 \%$ decrease in MZmt $2^{m и 290}$ mutant embryos (Fig. 4a). mt2 overexpression on the other hand led to a $27 \%$ increase in vegfc RNA levels in zebrafish embryos (Fig. 4a). To test whether vegfc transcripts are specifically affected, we analyzed further genes in $m t 2$-deficient and $m t 2$-overexpressing embryos. We chose flila as an EC-specific gene and myod1, a muscle-specific marker to represent other tissues [62]. We observed no significant changes in either flila or myodl transcript levels, irrespective of the $m t 2$ expression level. In contrast the significant changes in vegfc transcripts correlated with the changes in $m t 2$ expression as vegfc levels were decreased in $m t 2$-deficient 

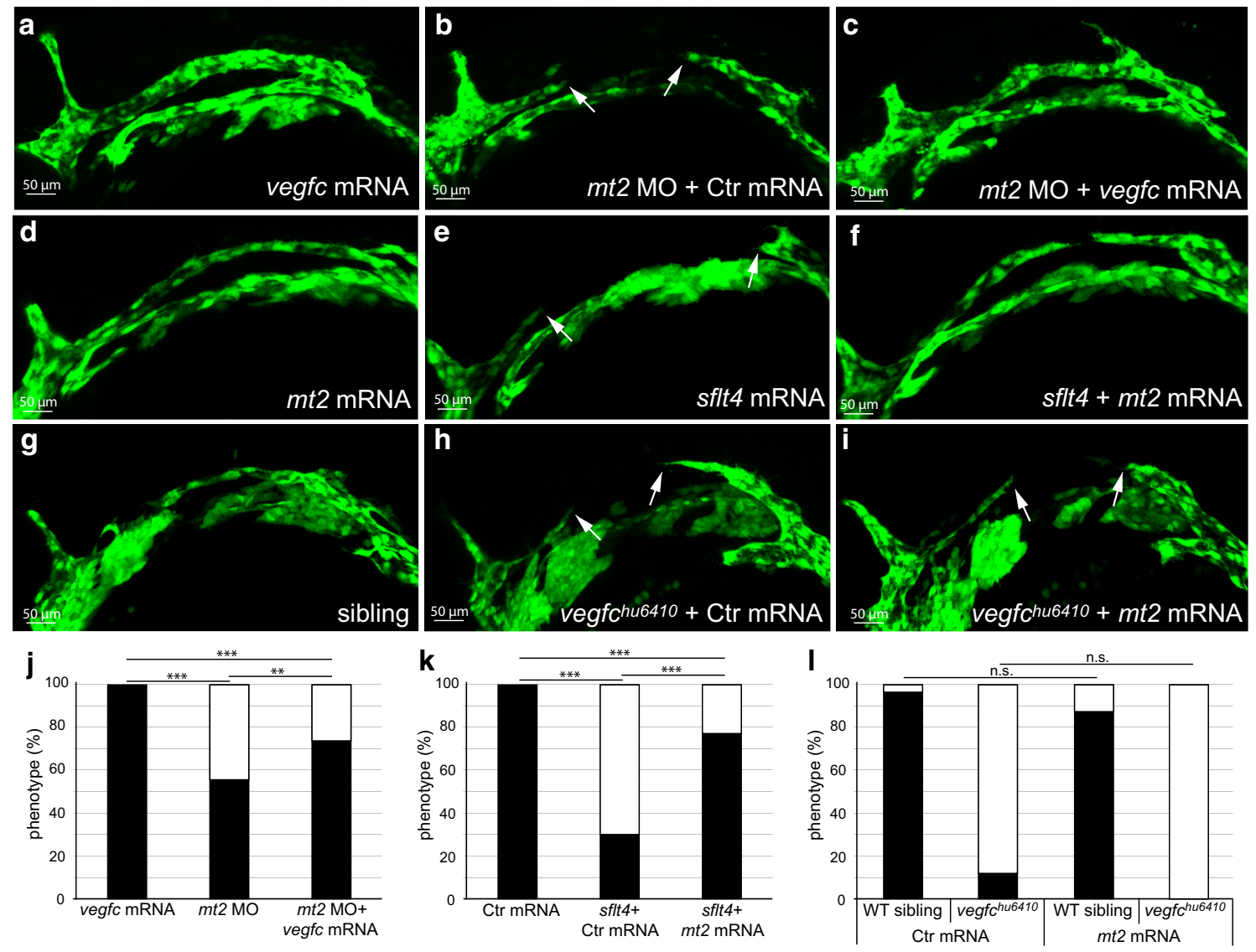

$\square$ PHBC existent $\square$ PHBC missing

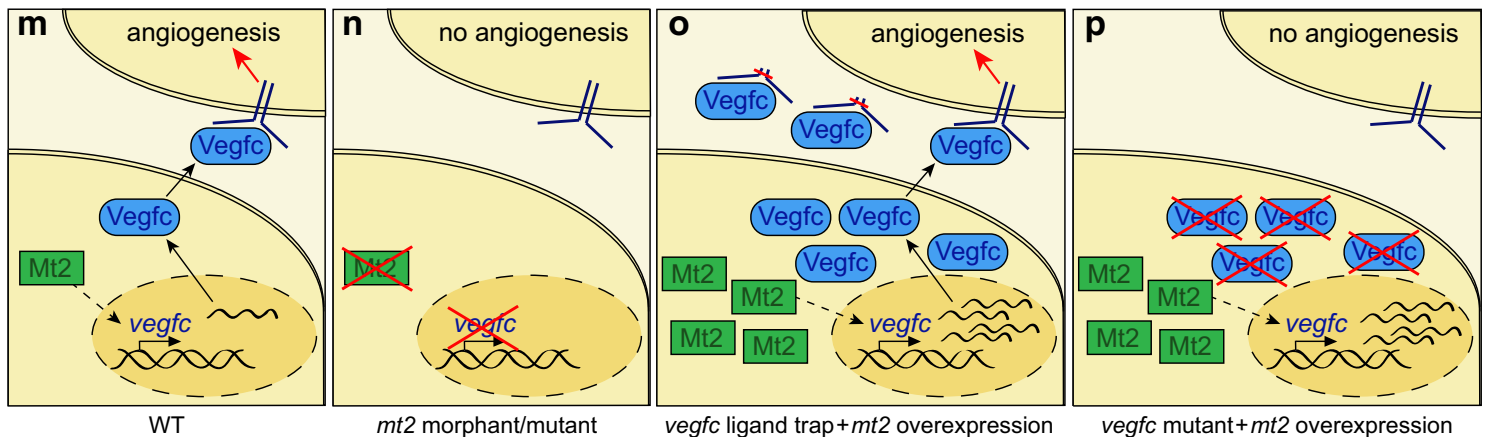

Fig. 3 Mt2 acts upstream of Vegfc in PHBC formation. a-c, j Injection of vegfc mRNA rescued $m t 2$ deficiency in $m t 2$ morphants. Overexpression of vegfc mRNA does not disturb PHBC formation (a). Upon $m t 2$ MO injection (b), $44 \%$ of the embryos lack the PHBC, while upon co-injection with vegfc mRNA (c) PHBC formation becomes rescued in half of the affected embryos (quantification of different experiments shown in $\mathbf{j}$ ). $\mathbf{d}-\mathbf{f}, \mathbf{k} m t 2$ overexpression rescued PHBC formation defects induced by overexpression of a Vegfc ligand trap ( $s f t 4$ overexpression). Injection of $m t 2 \mathrm{mRNA}$ resulted in normal PHBC development (d). Depletion of Vegfc through injection of sflt4 mRNA led to a failure in PHBC formation in $68 \%$ of the embryos (e). Co-injection of both $m t 2$ and sftt mRNA rescued PHBC formation and left only $23 \%$ of embryos showing no PHBC (quantifications of different experiments shown in $\mathbf{k}$ ). $\mathbf{g}-\mathbf{i}$, l Overexpression of $m t 2$ mRNA in embryos with a genetic null mutation in the vegfc gene (vegfc ${ }^{\text {hu6410}}$ ) could not rescue the PHBC phenotype. Embryos were scored for their phenotype and subsequently genotyped for the vegfc mutation (quantifications of different experiments shown in l). The analysis was performed using $T g(k d r l: E G F P)^{s 843}(\mathbf{a}-\mathbf{f})$ and $T g(f i l a: E G F P)^{y l}(\mathbf{g}-\mathbf{i})$ embryos. j$\mathbf{k}$ Quantifications of the phenotypes observed after injection of indicated reagents: Black bars label percent of embryos with the PHBC formed, white bars label percent of embryos lacking the PHBC. Statistical significance was calculated with the Chi-square test, $n=228 \quad(\mathbf{j}), \quad n=277 \quad(\mathbf{k}), \quad n=124 \quad(\mathbf{l}), \quad * * P<0.01$; $* * * P<0.001 ;$ n.s., not significant. $\mathbf{m}-\mathbf{p}$ Schematics representing the proposed mechanisms of angiogenesis regulation in the experiments shown in $\mathbf{a}-\mathbf{l}$ 


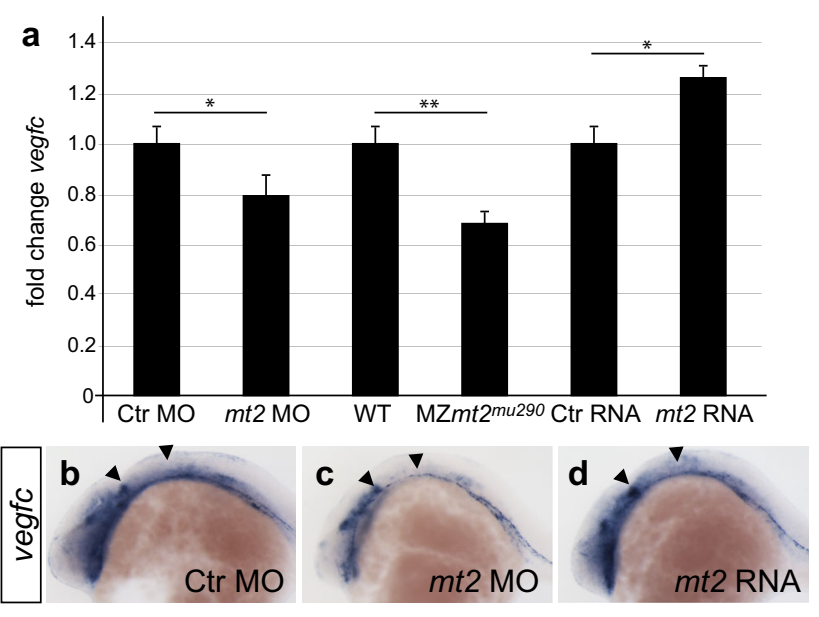

Fig. 4 Mt2 regulates transcript levels of vegfc. a $\mathrm{qPCR}$ analysis of vegfc transcript levels (a) in $m t 2$ morphants, MZmt $2^{m u 290}$ mutants and $m t 2$ mRNA-injected embryos compared to Ctr embryos. Both morphants and mutants showed a significant decrease in vegfc transcript levels, while overexpression of $m t 2$ led to an increase in vegfc transcripts. $n=3, * P<0.05 ; * * P<0.01$; error bars represent SEM; b-d in situ hybridization for vegfc in hpf embryos injected with $m t 2 \mathrm{MO}$ or with $m t 2$ mRNA showed a reduction in vegfc expression upon knockdown of $m t 2$ (c) and an increase after $m t 2$ mRNA injection $(\mathbf{d})$

and increased in $m t 2$-overexpressing cells (Fig S6). The analysis of vegfc RNA transcript levels via in situ hybridization showed similar results in some domains of $v e g f c$ expression (Fig. $4 \mathrm{~b}-\mathrm{d}$ ). $m t 2$ morphants show reduced $v e g f c$ staining, especially in the region, where the PHBCs develop (Fig. 4c, arrowheads). Interestingly, the increase in $v e g f c$ RNA expression in $m t 2$-injected embryos was also confined to specific domains, including the region of PHBC development (Fig. 4b-d, arrowheads), but not ubiquitously distributed (Fig. 4d). Therefore, our results show that Mt2 is required for regulating vegfc, e.g., during PHBC formation, but it is not sufficient to induce general vegfc expression ectopically. This can be further substantiated when comparing the WT expression patterns of vegfc and $m t 2$, showing that some domains of vegfc expression are in the same region as $m t 2$ expression, while there are also vegfc expression domains in areas not expressing high amounts of $m t 2$ (Fig. S6). We claim that the regulation of vegfc via Mt2 is specifically confined to specific vascular niches, such as the region of PHBC formation.

\section{Other metallothioneins cannot regulate vegfc expression}

To get more mechanistic insight how Mt2 could regulate $v e g f c$ expression, we questioned whether vegfc expression regulation could be a consequence of a cellular stress and hence would require the detoxifying features characteristic to all Metallothioneins (Mts). Therefore, we performed knockdown and overexpression experiments using another Metallothionein family member, metallothionein-B-like (mtbl; Fig. 5). To analyze $m t b l$-deficient embryos, we used again both translation and splice blocking MOs for our analysis and validated the functionality of the spbMO using RT-PCR (Fig. 5c). Even though $m t b l$ deficiency led to defective development of the CCVs and Ses (Fig. S7), $m t b l$ morphants showed normal PHBC development (Fig. 5b), indicating that during normal embryonic development Mt2 is specifically required for regulating vegfc expression. We next analyzed whether, as shown for Mt2, excess amounts of ectopic Mtbl could compensate for Vegfc ligand depletion by the ligand trap sftt . While injection of $s f t 4$ mRNA again provoked defective PHBC development (Fig. 5e), co-injection with $m t b l$ mRNA did not rescue this phenotype (Fig. 5f, g). Furthermore, vegfc transcripts were not significantly changed upon knockdown of $m t b l$ (Fig. 5h). These results indicate that the regulation of vegfc transcription by Mt 2 is not based on its Metallothionein characteristics and therefore not part of a cellular stress response, but rather represents an additional specific function of Mt2.

\section{Discussion}

In this study, we showed that Mt2 regulates developmental angiogenesis in zebrafish by regulating vegfc mRNA expression. Vegfc regulates EC migration as a chemoattractant, e.g., by guiding ECs in the PHBCs [9, 11], and indeed, we show that correct migration of the PHBCs was perturbed by deletion of $m t 2$. Additionally, Vegfc regulates EC proliferation [13], which was also perturbed in $m t 2-$ deficient zebrafish embryos.

We analyzed the role of Mt2 in zebrafish angiogenesis using MO-mediated Mt2 ablation as well as by using TALENs to introduce mutations in the zebrafish $m t 2$ gene. While we observed the same phenotypes in morphants as well as mutants, the phenotypes occurred at different frequencies between morphants and even between different hypothetical null mutants of $m t 2$ (see Table 1). Multiple mechanisms have been discussed to explain differences between mutant and morphant phenotypes: reinitiation at a downstream AUG or at an alternative start codon, exon skipping or the upregulation of other compensatory genes [63]. From the $m t 2$ sequence we can exclude reinitiation at a downstream AUG or exon skipping as potential mechanisms. We cannot predict whether there would be reinitiation at non-AUG start codons. However, we here provided a detailed analysis demonstrating that differences in mRNA stability, caused by NMD-mediated decay of the transcript, might account for the variability of the observed 

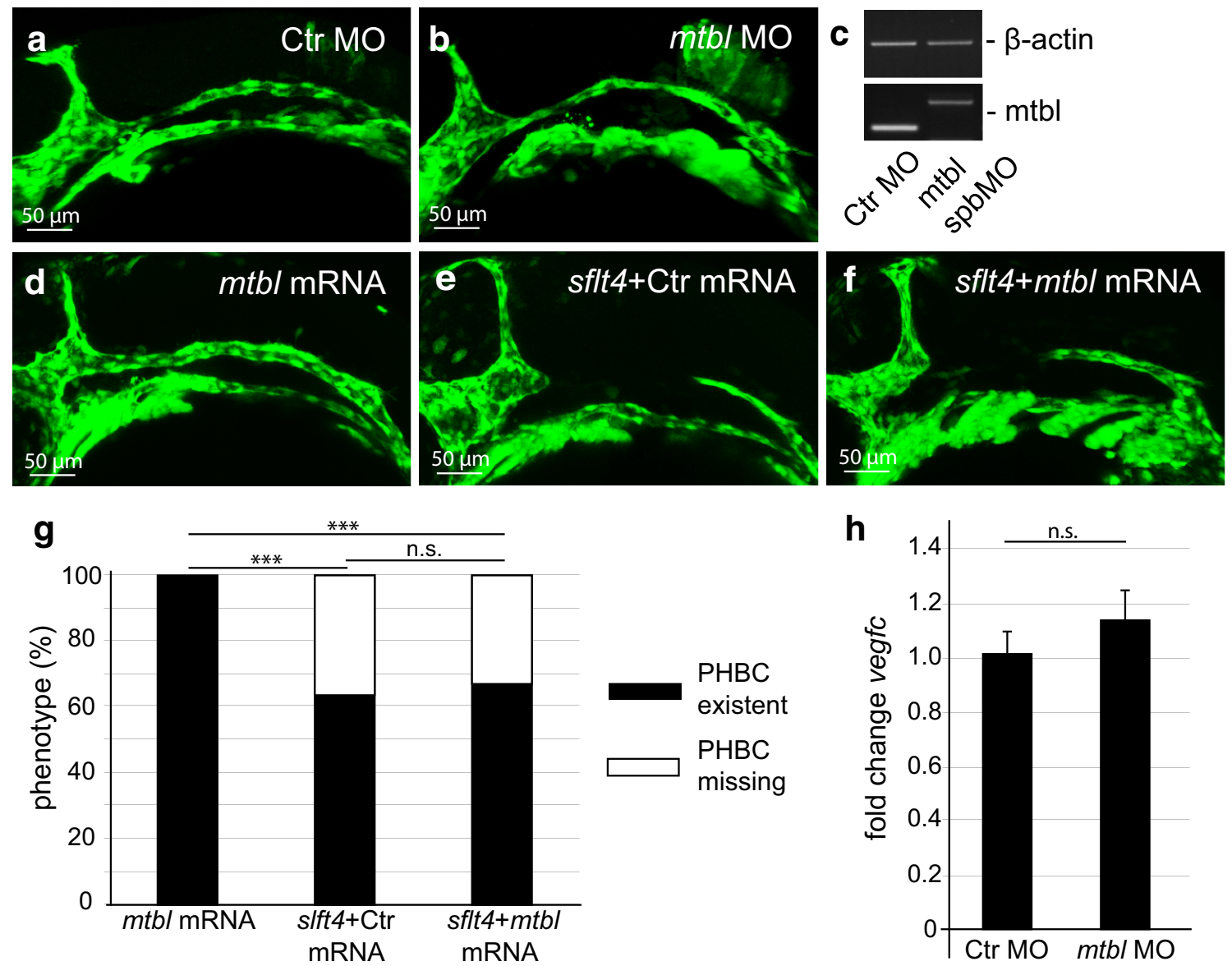

Fig. 5 The $m t 2$ paralogue metallothionein-B-like $(m t b l)$ does not regulate $\mathrm{PHBC}$ formation. $m t b l \mathrm{MO}$-mediated deficiency does not affect PHBC formation. $T g(k d r l: E G F P)^{s 843}$ embryos showed normal PHBC development at $24 \mathrm{hpf}$ after injection of Ctr MO (a) or $m t b l$ translation blocking MO (b). c Analysis of $m t b l$ splicing efficiency in embryos injected with Ctr MO or $m t b l$ spbMO. RT-PCR analysis showed a 327 bp amplicon in embryos injected with $m t b l$ spbMO, while functional splicing led to a $169 \mathrm{bp}$ amplicon in Ctr MO-injected embryos. $m t b l$ overexpression failed to rescue PHBC formation defects induced by overexpression of a Vegfc ligand trap (sflt

phenotypes. Our experiments show that a stronger efficiency of NMD led to a weaker penetrance of the phenotype, which might indicate transcript-level-based regulation of compensatory mechanisms in the embryo.

Additionally, even vegfc null mutants do not show full penetrance in failing to form the PHBC (Table 1, supplementary material [11]); therefore, embryos with a reduction in vegfc expression through $m t 2$ deficiency are not likely to display higher phenotypic frequencies.

In our study we identified a role for Mt2 in regulating angiogenesis upstream of transcriptional regulation of vegfc expression.

While in the zebrafish only two $m t$ genes exist, in mammals there are at least four different gene families with differentially expressed isoforms [64]. Analysis of the overexpression). Overexpression of $m t b l$ via mRNA injection (d) resulted in normal development of the PHBC, while sflt 4 mRNA injection resulted in PHBC formation failure (e). This phenotype could not be rescued through co-injection of $m t b l$ mRNA (f). Quantification comparing the percentages of embryos lacking the PHBC, with $n=350$, $* * * P<0.001$; n.s., not significant (g). The analysis was done with $T g(k d r l: E G F P)^{s 843}$ embryos. h qPCR analysis of $m t b l$ morphants showed no significant increase in the vegfc transcript. Error bars show SEM; n.s., not significant

amino acid sequence via UniProt revealed highest identity of the zebrafish Mt2 to the human and mouse MT1, closely followed by the human and mouse MT2. Mammalian Mt1 and $M t 2$ are supposedly very similar in their function [26] and have previously been implicated to be involved in angiogenic processes. The MZmt2 zebrafish knockout led to impaired development of major vessels, such as the PHBCs, the CCVs and the Ses and MZmt2-deficient embryos died during larval stages. The murine Mt1/2 double knockout in contrast was viable [36] and only displayed angiogenesis defects when challenged, e.g., by cortical freeze injury or femoral artery ligation [38-40]. As in zebrafish maternal message was capable of compensating $m t 2$ deficiency during embryonic angiogenesis, most likely in mammals other MT family might be able to 
compensate Mt1/2 deficiency during development. However, a link to angiogenesis has also been established for human Mts in vitro [65].

We demonstrated that $\mathrm{Mt} 2$ but not $\mathrm{Mtbl}$ regulates angiogenesis upstream of vegfc transcription. Mt family members are involved in regulating a large number of developmental processes, including cell survival, cell proliferation, migration, scavenging of reactive oxygen species, and modulating the immune response. Most of these capabilities have been attributed to the metal-binding capabilities, resulting, e.g., in removal of cofactor ions such as zinc [26, 30, 66]. The zebrafish Mtbl is capable of fulfilling these MT family member functions, but does not rescue $\mathrm{PHBC}$ development in $m t 2$ morphants or vegfc ligand reduced embryos. We present here the first evidence for an additional role of zebrafish $\mathrm{Mt} 2$ in regulating vegfc expression independent of $\mathrm{Mt}$ function. Interestingly, upregulation of different human MT isoforms was observed comparing the responses to physiological or hypoxic conditions [65]. This could be taken as an indication for differential regulatory functions of some human MT family proteins, independent of the functions common to all MTs.

We analyzed whether other transcript levels were regulated by zebrafish $\mathrm{Mt} 2$ in addition to vegfc. Neither the Vegfc regulator ccbel expression, nor the Pdgf/Vegf family member $c$-fos-induced growth factor (figf) expression was altered. In contrast, vegfa expression seemed also regulated downstream of MT2 (data not shown). Reduced Vegfa RNA [40] and VEGFA protein levels [38] were reported in Mtl/ 2-deficient mice. While changes in Vegfc expression explained the $\mathrm{PHBC}$ and $\mathrm{CCV}$ phenotypes, reduction in Vegfa expression would account for the failures in Se formation, as deficiency in either Vegfa or its receptor Kdrl result in severe Se phenotypes [9, 67].

In sum, we have identified a novel role of MT2 in regulating angiogenesis by regulating vegfc transcription, which might be conserved in mammals.

We for the first time show that this regulatory role is specific to zebrafish $\mathrm{Mt} 2$ and represents a novel, noncanonical function of MT2, most likely not attributed to metal-binding capabilities of MT proteins.

Acknowledgments We thank Mailin Hamm, Didier Stainier and Friedemann Kiefer for critical comments on the manuscript. We are grateful to Reinhild Bussmann for excellent fish husbandry and to Katja Müller for technical assistance. We would like to thank Stefan Volkery for imaging advice and microscope maintenance. This work was supported by the Northrhine Westphalia (NRW) "return fellowship" awarded to W. H. and by the Cells-in-Motion Cluster of Excellence (CiM-EXC 1003), Muenster, Germany (Project FF2013-14).

Conflict of interest The authors declare that they have no conflict of interest.
Ethical standard All applicable international, national and/or institutional guidelines for the care and use of animals were followed.

Open Access This article is distributed under the terms of the Creative Commons Attribution 4.0 International License (http://creativecommons.org/licenses/by/4.0/), which permits unrestricted use, distribution, and reproduction in any medium, provided you give appropriate credit to the original author(s) and the source, provide a link to the Creative Commons license, and indicate if changes were made.

\section{References}

1. Bussmann J, Lawson N, Zon L, Schulte-Merker S (2008) Zebrafish VEGF receptors: a guideline to nomenclature. PLoS Genet 4(5):e1000064. doi:10.1371/journal.pgen.1000064

2. Lohela M, Bry M, Tammela T, Alitalo K (2009) VEGFs and receptors involved in angiogenesis versus lymphangiogenesis. Curr Opin Cell Biol 21(2):154-165. doi:10.1016/j.ceb.2008.12.012

3. Shibuya M (2013) Vascular endothelial growth factor and its receptor system: physiological functions in angiogenesis and pathological roles in various diseases. J Biochem 153(1):13-19. doi: $10.1093 / \mathrm{Jb} / \mathrm{Mvs} 136$

4. Ferrara N (1999) Role of vascular endothelial growth factor in the regulation of angiogenesis. Kidney Int 56(3):794-814. doi:10. 1046/j.1523-1755.1999.00610.x

5. Gerhardt $\mathrm{H}$ (2008) VEGF and endothelial guidance in angiogenic sprouting. Organogenesis 4(4):241-246

6. Kuchler AM, Gjini E, Peterson-Maduro J, Cancilla B, Wolburg H, Schulte-Merker S (2006) Development of the zebrafish lymphatic system requires VEGFC signaling. Curr Biol 16(12):1244-1248. doi:10.1016/j.cub.2006.05.026

7. Tammela T, Alitalo K (2010) Lymphangiogenesis: molecular mechanisms and future promise. Cell 140(4):460-476. doi:10. 1016/J.Cell.01.045

8. Kukk E, Lymboussaki A, Taira S, Kaipainen A, Jeltsch M, Joukov V, Alitalo K (1996) VEGF-C receptor binding and pattern of expression with VEGFR-3 suggests a role in lymphatic vascular development. Development 122(12):3829-3837

9. Hogan BM, Herpers R, Witte M, Helotera H, Alitalo K, Duckers HJ, Schulte-Merker S (2009) Vegfc/Flt4 signalling is suppressed by Dll4 in developing zebrafish intersegmental arteries. Development 136(23):4001-4009. doi:10.1242/dev.039990

10. Dumont DJ, Jussila L, Taipale J, Lymboussaki A, Mustonen T, Pajusola K, Breitman M, Alitalo K (1998) Cardiovascular failure in mouse embryos deficient in VEGF receptor-3. Science 282(5390):946-949. doi:10.1126/Science.282.5390.946

11. Villefranc JA, Nicoli S, Bentley K, Jeltsch M, Zarkada G, Moore JC, Gerhardt H, Alitalo K, Lawson ND (2013) A truncation allele in vascular endothelial growth factor c reveals distinct modes of signaling during lymphatic and vascular development. Development 140(7):1497-1506. doi:10.1242/dev.084152

12. Karkkainen MJ, Haiko P, Sainio K, Partanen J, Taipale J, Petrova TV, Jeltsch M, Jackson DG, Talikka M, Rauvala H, Betsholtz C, Alitalo K (2004) Vascular endothelial growth factor $C$ is required for sprouting of the first lymphatic vessels from embryonic veins. Nat Immunol 5(1):74-80. doi:10.1038/ni1013

13. Helker CS, Schuermann A, Karpanen T, Zeuschner D, Belting HG, Affolter M, Schulte-Merker S, Herzog W (2013) The zebrafish common cardinal veins develop by a novel mechanism: lumen ensheathment. Development 140(13):2776-2786. doi:10. 1242/dev.091876 
14. Christiansen A, Detmar M (2011) Lymphangiogenesis and cancer. Genes Cancer 2(12):1146-1158. doi:10.1177/19476019 11423028

15. Moens S, Goveia J, Stapor PC, Cantelmo AR, Carmeliet P (2014) The multifaceted activity of VEGF in angiogenesis-implications for therapy responses. Cytokine Growth Factor Rev 25(4): 473-482. doi:10.1016/j.cytogfr.2014.07.009

16. Ristimaki A, Narko K, Enholm B, Joukov V, Alitalo K (1998) Proinflammatory cytokines regulate expression of the lymphatic endothelial mitogen vascular endothelial growth factor-C. J Biol Chem 273(14):8413-8418. doi:10.1074/Jbc.273.14.8413

17. Enholm B, Paavonen K, Ristimaki A, Kumar V, Gunji Y, Klefstrom J, Kivinen L, Laiho M, Olofsson B, Joukov V, Eriksson U, Alitalo K (1997) Comparison of VEGF, VEGF-B, VEGF-C and Ang-1 mRNA regulation by serum, growth factors, oncoproteins and hypoxia. Oncogene 14(20):2475-2483. doi:10.1038/Sj.Onc. 1201090

18. Gunningham SP, Currie MJ, Han C, Turner K, Scott PAE, Robinson BA, Harris AL, Fox SB (2001) Vascular endothelial growth factor-B and vascular endothelial growth factor-C expression in renal cell carcinomas: regulation by the von Hippel-Lindau gene and hypoxia. Cancer Res 61(7):3206-3211

19. Schuermann A, Helker CSM, Herzog W (2014) Angiogenesis in zebrafish. Semin Cell Dev Biol 31:106-114. doi:10.1016/J. Semcdb.04.037

20. Isogai S, Horiguchi M, Weinstein BM (2001) The vascular anatomy of the developing zebrafish: an atlas of embryonic and early larval development. Dev Biol 230(2):278-301. doi:10.1006/ dbio.2000.9995

21. Walls JR, Coultas L, Rossant J, Henkelman RM (2008) Threedimensional analysis of vascular development in the mouse embryo. PLoS One 3(8):e2853. doi:10.1371/journal.pone.000 2853

22. Ellertsdottir E, Lenard A, Blum Y, Krudewig A, Herwig L, Affolter M, Belting HG (2010) Vascular morphogenesis in the zebrafish embryo. Dev Biol 341(1):56-65. doi:10.1016/j.ydbio. 2009.10.035

23. Gore AV, Monzo K, Cha YR, Pan W, Weinstein BM (2012) Vascular development in the zebrafish. Cold Spring Harb Perspect Med 2(5):a006684. doi:10.1101/cshperspect.a006684

24. Auer TO, Del Bene F (2014) CRISPR/Cas9 and TALEN-mediated knock-in approaches in zebrafish. Methods 69(2):142-150. doi:10.1016/j.ymeth.2014.03.027

25. Bedell VM, Wang Y, Campbell JM, Poshusta TL, Starker CG, Krug RG, Tan WF, Penheiter SG, Ma AC, Leung AYH, Fahrenkrug SC, Carlson DF, Voytas DF, Clark KJ, Essner JJ, Ekker SC (2012) In vivo genome editing using a high-efficiency TALEN system. Nature 491(7422):114-118. doi:10.1038/ Nature 1537

26. Nielsen AE, Bohr A, Penkowa M (2007) The balance between life and death of cells: roles of metallothioneins. Biomark Insights 1:99-111

27. Pedersen MO, Larsen A, Stoltenberg M, Penkowa M (2009) The role of metallothionein in oncogenesis and cancer prognosis. Prog Histochem Cytochem 44(1):29-64. doi:10.1016/J.Proghi.10.001

28. Wu SM, Tsai PR, Yan CJ (2012) Maternal cadmium exposure induces $\mathrm{mt} 2$ and $\mathrm{smtB}$ mRNA expression in zebrafish (Danio rerio) females and their offspring. Comp Biochem Physiol C Toxicol Pharmacol 156(1):1-6. doi:10.1016/J.Cbpc.02.001

29. Wu SM, Zheng YD, Kuo CH (2008) Expression of mt2 and smt$\mathrm{B}$ upon cadmium exposure and cold shock in zebrafish (Danio rerio). Comp Biochem Physiology C Toxicol Pharmacol 148(2):184-193. doi:10.1016/J.Cbpc.05.007

30. Vasak M, Meloni G (2011) Chemistry and biology of mammalian metallothioneins. J Biol Inorg Chem 16(7):1067-1078. doi:10. 1007/S00775-011-0799-2
31. Yan CHM, Chan KM (2004) Cloning of zebrafish metallothionein gene and characterization of its gene promoter region in HepG2 cell line. Biochim Et Biophys Acta-Gene Struct Expr 1679(1):47-58. doi:10.1016/J.Bbaexp.04.004

32. Fu J, Lv HJ, Guan HX, Ma XY, Ji MJ, He NY, Shi BY, Hou P (2013) Metallothionein 1G functions as a tumor suppressor in thyroid cancer through modulating the PI3 K/Akt signaling pathway. BMC Cancer 13:462. doi:10.1186/1471-2407-13-462

33. Murakami S, Miyazaki I, Sogawa N, Miyoshi K, Asanuma M (2014) neuroprotective Effects of metallothionein against rotenone-induced myenteric Neurodegeneration in parkinsonian mice. Neurotox Res 26(3):285-298. doi:10.1007/S12640-0149480-1

34. Xue WL, Liu YL, Zhao JC, Cai L, Li XK, Feng WK (2012) Activation of HIF-1 by metallothionein contributes to cardiac protection in the diabetic heart. Am J Physiol Heart Circ Physiol 302(12):H2528-H2535. doi:10.1152/Ajpheart.00850.2011

35. Zhou GH, Li XK, Hein DW, Xiang XL, Marshall JP, Prabhu SD, Cai L (2008) Metallothionein suppresses angiotensin II-induced nicotinamide adenine dinucleotide phosphate oxidase activation, nitrosative stress, apoptosis, and pathological remodeling in the diabetic heart. J Am Coll Cardiol 52(8):655-666. doi:10.1016/J. Jacc.05.019

36. Masters BA, Kelly EJ, Quaife CJ, Brinster RL, Palmiter RD (1994) Targeted disruption of metallothionein-I and metallothionein-Ii genes increases sensitivity to cadmium. Proc Natl Acad Sci USA 91(2):584-588. doi:10.1073/Pnas.91.2.584

37. Michalska AE, Choo KHA (1993) Targeting and germ-line transmission of a null mutation at the metallothionein I-loci and Ii-loci in Mouse. Proc Natl Acad Sci USA 90(17):8088-8092. doi:10.1073/Pnas.90.17.8088

38. Penkowa M, Carrasco J, Giralt M, Molinero A, Hernandez J, Campbell IL, Hidalgo J (2000) Altered central nervous system cytokine-growth factor expression profiles and angiogenesis in metallothionein-I + II deficient mice. J Cereb Blood Flow Metab 20(8):1174-1189. doi:10.1097/00004647-200008000-00003

39. Penkowa M, Carrasco J, Giralt M, Moos T, Hidalgo J (1999) CNS wound healing is severely depressed in metallothionein I and II-deficient mice. J Neurosci 19(7):2535-2545

40. Zbinden S, Wang JS, Adenika R, Schmidt M, Tilan JU, Najafi AH, Peng XZ, Lassance-Soares RM, Iantorno M, Morsli H, Gercenshtein L, Jang GJ, Epstein SE, Burnett MS (2010) Metallothionein enhances angiogenesis and arteriogenesis by modulating smooth muscle cell and macrophage function. Arterioscler Thromb Vasc Biol 30(3):477-482. doi:10.1161/Atvbaha. 109.200949

41. Palmiter RD (1995) Constitutive expression of metallothioneinIii (Mt-Iii), but not Mt-I, inhibits growth when cells become zincdeficient. Toxicol Appl Pharmacol 135(1):139-146. doi:10.1006/ Taap.1995.1216

42. Smith E, Drew PA, Tian ZQ, De Young NJ, Liu JF, Mayne GC, Ruszkiewicz AR, Watson DI, Jamieson GG (2005) Metallothionien 3 expression is frequently down-regulated in oesophageal squamous cell carcinoma by DNA methylation. Mol Cancer 4:42. doi:10.1186/1476-4598-4-42

43. Carrasco J, Penkowa M, Giralt M, Camats J, Molinero A, Campbell IL, Palmiter RD, Hidalgo J (2003) Role of metallothionein-III following central nervous system damage. Neurobiol Dis 13(1):22-36. doi:10.1016/S0969-9961(03)00015-9

44. Tio L, Villarreal L, Atrian S, Capdevila M (2004) Functional differentiation in the mammalian metallothionein gene familymetal binding features of mouse MT4 and comparison with its paralog MT1. J Biol Chem 279(23):24403-24413. doi:10.1074/ Jbc.M401346200

45. Westerfield M (1993) The zebrafish book. University of Oregon press, Eugene 
46. Jin SW, Beis D, Mitchell T, Chen JN, Stainier DY (2005) Cellular and molecular analyses of vascular tube and lumen formation in zebrafish. Development 132(23):5199-5209. doi:10.1242/ dev.02087

47. Lawson ND, Weinstein BM (2002) In vivo imaging of embryonic vascular development using transgenic zebrafish. Dev Biol 248(2):307-318. doi:10.1006/dbio.2002.0711

48. Roman BL, Pham V, Lawson ND, Kulik M, Childs S, Lekven AC, Garrity DM, Moon RT, Fishman MC, Lechleider RJ, Weinstein BM (2002) Disruption of acvrl1 increases endothelial cell number in zebrafish cranial vessels. Development 129(12):3009-3019 Dev14536

49. Le Guen L, Karpanen T, Schulte D, Harris NC, Koltowska K, Roukens G, Bower NI, van Impel A, Stacker SA, Achen MG, Schulte-Merker S, Hogan BM (2014) Ccbe1 regulates Vegfcmediated induction of Vegfr3 signaling during embryonic lymphangiogenesis. Development 141(6):1239-1249. doi:10.1242/ dev.100495

50. Cermak T, Doyle EL, Christian M, Wang L, Zhang Y, Schmidt C, Baller JA, Somia NV, Bogdanove AJ, Voytas DF (2011) Efficient design and assembly of custom TALEN and other TAL effector-based constructs for DNA targeting. Nucleic Acids Res 39(12):e82. doi:10.1093/nar/gkr218

51. Wittkopp N, Huntzinger E, Weiler C, Sauliere J, Schmidt S, Sonawane M, Izaurralde E (2009) Nonsense-mediated mrna decay effectors are essential for zebrafish embryonic development and survival. Mol Cell Biol 29(13):3517-3528. doi:10. 1128/Mcb.00177-09

52. Santoro MM, Samuel T, Mitchell T, Reed JC, Stainier DY (2007) Birc2 (cIap1) regulates endothelial cell integrity and blood vessel homeostasis. Nat Genet 39(11):1397-1402. doi:10.1038/ng.2007. 8

53. Hogan BM, Bos FL, Bussmann J, Witte M, Chi NC, Duckers HJ, Schulte-Merker S (2009) Ccbe1 is required for embryonic lymphangiogenesis and venous sprouting. Nat Genet 41(4):396-398. doi:10.1038/ng.321

54. Makinen T, Jussila L, Veikkola T, Karpanen T, Kettunen MI, Pulkkanen KJ, Kauppinen R, Jackson DG, Kubo H, Nishikawa S, Yla-Herttuala S, Alitalo K (2001) Inhibition of lymphangiogenesis with resulting lymphedema in transgenic mice expressing soluble VEGF receptor-3. Nat Med 7(2):199-205. doi:10.1038/ 84651

55. Thisse C, Thisse B (2008) High-resolution in situ hybridization to whole-mount zebrafish embryos. Nat Protoc 3(1):59-69. doi:10. 1038/nprot.2007.514

56. Helker CS, Schuermann A, Pollmann C, Chng SC, Kiefer F, Reversade B, Herzog W (2015) The hormonal peptide Elabela guides angioblasts to the midline during vasculogenesis. Elife. doi:10.7554/eLife.06726

57. Herzog W, Muller K, Huisken J, Stainier DY (2009) Genetic evidence for a noncanonical function of seryl-tRNA synthetase in vascular development. Circ Res 104(11):1260-1266. doi:10. 1161/CIRCRESAHA.108.191718

58. Robu ME, Larson JD, Nasevicius A, Beiraghi S, Brenner C, Farber SA, Ekker SC (2007) p53 activation by knockdown technologies. PLoS Genet 3(5):e78. doi:10.1371/journal.pgen. 0030078

59. Law SHW, Sargent TD (2014) The serine-threonine protein kinase PAK4 Is dispensable in zebrafish: identification of a morpholino-generated pseudophenotype. PLoS One 9(6):e100268. doi:10.1371/journal.pone.0100268

60. Schulte-Merker S, Stainier DYR (2014) Out with the old, in with the new: reassessing morpholino knockdowns in light of genome editing technology. Development 141(16):3103-3104. doi:10. 1242/Dev.112003

61. Chen WY, John JA, Lin CH, Lin HF, Wu SC, Lin CH, Chang CY (2004) Expression of metallothionein gene during embryonic and early larval development in zebrafish. Aquat Toxicol 69(3):215-227. doi:10.1016/j.aquatox.2004.05.004

62. Musso G, Tasan M, Mosimann C, Beaver JE, Plovie E, Carr LA, Chua HN, Dunham J, Zuberi K, Rodriguez H, Morris Q, Zon L, Roth FP, MacRae CA (2014) Novel cardiovascular gene functions revealed via systematic phenotype prediction in zebrafish. Development 141(1):224-235. doi:10.1242/Dev.099796

63. Stainier DYR, Kontarakis Z, Rossi A (2015) Making sense of anti-sense. Data Dev Cell 32(1):7-8. doi:10.1016/J.Devcel.12. 012

64. Seren N, Glaberman S, Carretero MA, Chiari Y (2014) Molecular evolution and functional divergence of the metallothionein gene family in vertebrates. J Mol Evol 78(3-4):217-233. doi:10.1007/ s00239-014-9612-5

65. Schulkens IA, Castricum KC, Weijers EM, Koolwijk P, Griffioen AW, Thijssen VL (2014) Expression, regulation and function of human metallothioneins in endothelial cells. J Vasc Res 51(3):231-238. doi:10.1159/000365550

66. Carpene E, Andreani G, Isani G (2007) Metallothionein functions and structural characteristics. J Trace Elem Med Biol 21(Suppl 1):35-39. doi:10.1016/j.jtemb.2007.09.011

67. Covassin LD, Siekmann AF, Kacergis MC, Laver E, Moore JC, Villefranc JA, Weinstein BM, Lawson ND (2009) A genetic screen for vascular mutants in zebrafish reveals dynamic roles for Vegf/Plcg1 signaling during artery development. Dev Biol 329(2):212-226. doi:10.1016/j.ydbio.2009.02.031 